Article

\title{
Characterization of Core-Shell Spherical Lens for Microtracking Concentrator Photovoltaic System
}

\author{
Masakazu Nakatani ${ }^{1,2, *(\mathbb{D})}$ and Noboru Yamada ${ }^{1}$ (D) \\ 1 Department of Energy and Environment Science, Nagaoka University of Technology, \\ 1603-1 Kamitomioka-machi, Nagaoka 940-2188, Japan; noboru@nagaokaut.ac.jp \\ 2 Sun Marion Co., Ltd., 1603-1 Kamitomioka-machi, Nagaoka 940-2188, Japan \\ * Correspondence: nakatani@sun-marion.com
}

Received: 16 July 2019; Accepted: 3 September 2019; Published: 12 September 2019

check for updates

\begin{abstract}
The optical characteristics of a radially symmetrical core-shell spherical (CSSP) lens is analyzed for its suitability to application in microtracking concentrator photovoltaic systems (MTCPVs). The CSSP lens is compared to a conventional homogenous spherical lens through both ray-tracing simulations and outdoor experiments. Simulation results show that the CSSP lens is superior to the conventional homogenous spherical lens in terms of its optical efficiency for long focal lengths, for which the CSSP lens exhibits less spherical and chromatic aberrations. Outdoor experiments are conducted using test concentrator photovoltaic (CPV) modules with prototype CSSP and homogenous spherical lenses; the trend of the measured short circuit current agrees with the that of the simulated optical efficiency for both lenses. Furthermore, compared to the homogenous lens, the CSSP lens significantly increases module efficiency because of its better illumination uniformity at the solar cell surface. The optical characteristics of the CSSP lens are preferable for MTCPVs with a spherical lens array to achieve a higher module efficiency for a wider incidence angle although further studies on more practical system configurations are needed.
\end{abstract}

Keywords: photovoltaics; solar concentrators; solar cells; geometrical optics; concentrator photovoltaics; spherical lens

\section{Introduction}

Multijunction solar cells can achieve a high solar-cell efficiency of 46\% [1]. Currently, the maximum efficiency of a concentrator photovoltaic (CPV) system that includes a multijunction solar cell and a concentrator is $36.7 \%$ [2]. Although a high-concentration CPV system has high solar-cell efficiency, it requires a solar tracking system. Pedestal solar trackers are commonly used in CPV systems; however, pedestal trackers are massive and unsuitable for building integrated photovoltaics (BIPV).

A microtracking concentrator photovoltaic system (MTCPV) that integrates a CPV and a solar tracker into a module is being developed [3]. Examples of MTCPV structures include (a) a two-lens structure on the top surface of a multijunction solar cell [4], (b) an upper waveguide and lens structure on a multijunction solar cell [5-11], (c) a structure combining an upside lens and a downside mirror on a multijunction solar cell [12,13], (d) a resin filled mirror under a solar cell stage [14], (e) a structure comprised of a wide angle aplanatic lens and a solar cell stage with three-dimensional control [15], and (f) a structure comprised of a gradient-index(GRIN) lens $[16,17]$ and a solar cell stage with three-dimensional control [18]. Of these, structure (f) has yet to be experimentally demonstrated as it requires a gradient low-refractive index (RI) structure. The $\mathrm{RI}$ of $\mathrm{Mg}_{2} \mathrm{~F}$, a conventional transparent low-RI material, is 1.39 [19]. Optical porous materials can achieve a lower RI [20]; however, they are still under development for practical use [21,22]. These reported MTCPV systems have demonstrated 
the technological potential of the MTCPV; however, further investigation is still needed to develop a practical optical system with better performance and a simpler structure.

Previously, we had proposed a spherical-lens microtracking CPV (SMTCPV) system [23]. This proposed SMTCPV system requires a spherical lens with a long focal length and a low spherical aberration. A radially symmetrical core-shell spherical (CSSP) lens has been studied for use in an antenna as well as in a CPV [24,25]. The CSSP lens is a kind of a simplified GRIN lens; because of the low-RI material used as the core material, it has a low spherical aberration and a long focal length. The CSSP lens may be suitable for application in a SMTCPV system. However, experimental studies of the SMTCPV with the CSSP lens have not been reported to date. Accordingly, here we analyze the optical characteristics of a CSSP lens made of commercially available materials, fabricate a prototype, evaluate the real-world performance, and compare its performance with a conventional homogenous spherical lens.

\section{Configuration of the SMTCPV System}

Figure 1 depicts the tracking motion of a SMTCPV system consisting of a spherical lens array and a solar-cell stage. Sunlight is concentrated onto the solar cell via mechanically controlling the relative position of the lens array and the solar-cell stage. As an example, the solar-cell stage can move in the lateral direction (XY movement), while the spherical-lens array moves in the vertical direction (Z movement), thus enabling a wide acceptance angle. Unlike a hemisphere or other lenses, the optical performance of the spherical lens does not depend on the angle of incidence unless interferences by adjacent lenses occurs in an array configuration. Thus, changing the lens angle, i.e., rotating the lens, is unnecessary, and the focal position is easily known, contributing to a simpler mechanical design and tracking control. A longer focal length is desirable for an SMTCPV system because the longer the focal length, the wider the mechanical angle limit. Figure 2 shows the schematic of the mechanical angle limit $\theta_{\text {limit }}$, which is defined as the angle at which the top end surface of the solar-cell stage contacts the bottom end surface of the spherical lens. Thus, a longer focal length $f$ enables a wider $\theta_{\text {limit, }}$ and contributes to widening the acceptance angle. Additionally, a shorter focal length would increase the reflection loss at the solar cell surface as the solar cell must receive the concentrated rays at a high incidence angle. If a micro spherical lens array is integrated with a compact internal tracking mechanism, a compact SMTCPV system can be designed. When a spherical lens with a diameter of 3-7 cm is used, the expected module thickness is $10-20 \mathrm{~cm}$. Here, the optical performance of a single CSSP lens is examined as a first-step in considering its application to the SMTCPV system.
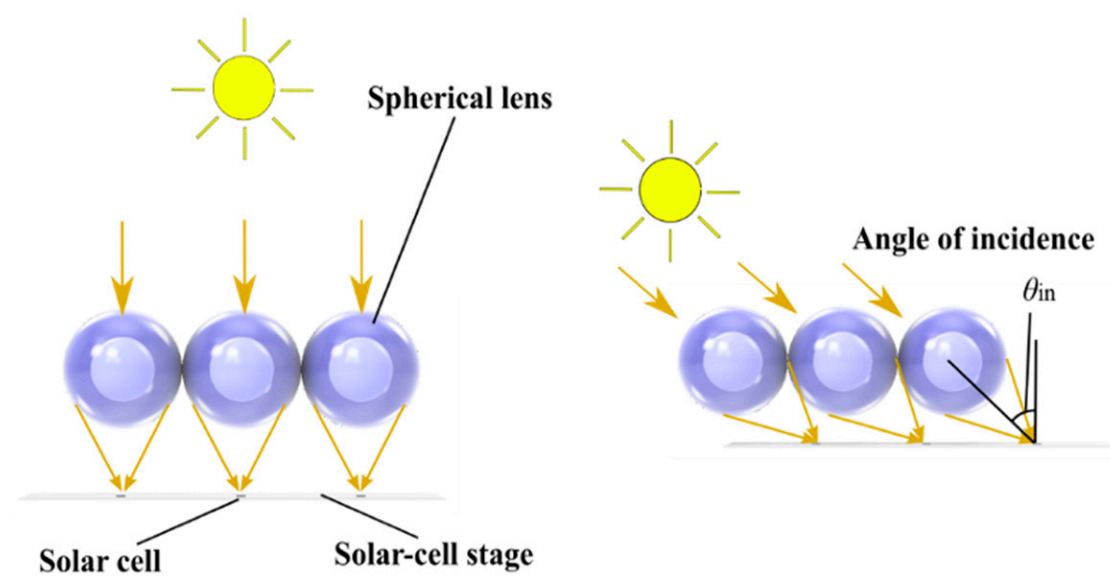

Figure 1. Tracking motion of the spherical-lens microtracking concentrator photovoltaic (CPV) (SMTCPV). 




Figure 2. Mechanical angle limit of the microtracking concentrator photovoltaic systems (MTCPV) with a spherical lens.

\section{Simulation}

To comprehend the optical characteristics of the CSSP lens, ray-tracing analyses are conducted in the following steps: (1) Analysis of monochromatic light (sodium D line) without considering volumetric absorption by lens materials; (2) analysis of chromatic light (nearly full solar spectrum) without considering volumetric absorption by lens materials; (3) analysis of chromatic light (nearly full solar spectrum) with considerations of volumetric absorption by lens materials.

\subsection{CSSP Lenses Characteristics for Monochromatic Light without Lens Material Absorption}

Figure 3 shows the simulation model of the CSSP lens. Table 1 lists the simulation conditions A, which includes the specifications of the CSSP lens and the homogenous spherical lens, for comparison. This CSSP lens model includes a shell comprised of solid plastic (PMMA) and a core composed of liquid silicone oil. Three-dimensional ray-tracing was performed using commercial software (LightTools 8.7.0), in which the Fresnel reflection loss was considered. In this subsection, volumetric absorption was ignored.

The core RI, $n_{\text {core }}=1.403$, is a silicone oil RI (Shin-Etsu Silicone KF-96-500cs [26]) at $589.5 \mathrm{~nm}$. The core material requires the conditions listed in Table 2, which are satisfied by KF-96-500cs. The CSSP lens requires two different materials with different RIs. In the solid state, most materials have different thermal expansion coefficients and tend to cause a separation at the core-shell interface. Thus, one solution is to use a material in the liquid state with a high transparency, low RI, and less evaporation. The shell RI, $n_{\text {shell }}=1.491$, is a PMMA RI at $587.6 \mathrm{~nm}$ [27]. The core radius, $r_{\text {core, }}$ at which spherical aberration was effectively reduced was determined to be $10.2 \mathrm{~mm}$ [23]. For comparison, two homogenous spherical lenses composed of only PMMA and only KF-96-500cs are also simulated. The KF-96-500cs homogeneous spherical lens is a hypothetical lens since it is liquid. As explained in the Section 2, a higher optical efficiency and longer focal length are preferable for the SMTCPV system; thus, these two factors were analyzed.

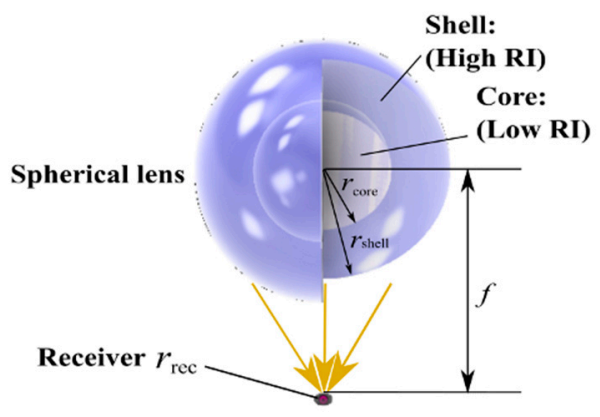

Figure 3. Simulation model of the core-shell spherical (CSSP) lens. 
Table 1. Simulation conditions A.

\begin{tabular}{|c|c|c|c|}
\hline Parameters & $\begin{array}{l}\text { PMMA Homogenous } \\
\text { Spherical Lens }\end{array}$ & $\begin{array}{l}\text { KF-96-500cs } \\
\text { Homogeneous } \\
\text { Spherical Lens }\end{array}$ & CSSP Lens \\
\hline $\begin{array}{l}\text { Shell refractive index } \\
\text { (RI), } n_{\text {shell }}\end{array}$ & $1.491 @ 589$ nm & $1.403 @ 589$ nm & $1.491 @ 589$ nm \\
\hline Core RI, $n_{\text {core }}$ & N/A & N/A & $1.403 @ 589$ nm \\
\hline Shell radius, $r_{\text {shell }}$ & $15 \mathrm{~mm}$ & $15 \mathrm{~mm}$ & $15 \mathrm{~mm}$ \\
\hline Core radius, $r_{\text {core }}$ & N/A & N/A & $10.2 \mathrm{~mm}\left(0.68 \times r_{\text {shell }}\right)$ \\
\hline $\begin{array}{c}\text { Geometrical } \\
\text { concentration ratio, } C\end{array}$ & & $\begin{array}{c}6.25 \times \text { for Figure } 8 a \\
100 \times \text { for Figures } 8 b \text { and } 9\end{array}$ & \\
\hline $\begin{array}{c}\text { Ray spectrum } \\
\text { Absorption by lens } \\
\text { materials }\end{array}$ & & $\begin{array}{l}\text { Sodium D line }(589 \mathrm{~nm}) \\
\text { Ignored }\end{array}$ & \\
\hline
\end{tabular}

Table 2. Core material requirements and KF-96-500 cs specifications.

\begin{tabular}{|c|c|c|}
\hline Required & Reason & KF-96-500cs [26] \\
\hline Low refractive index & For long focal length & $1.403 @ 589$ nm \\
\hline Liquid in ambient & $\begin{array}{l}\text { Solid material tends to cause a } \\
\text { separation at core-shell interface }\end{array}$ & Liquid \\
\hline Low volatile matter content & No bubble formation & $\max 0.5 \%$ at $150{ }^{\circ} \mathrm{C} / 24 \mathrm{~h}$ \\
\hline
\end{tabular}

Figure 4 shows the ray behavior of both the homogenous and the CSSP lenses, wherein Figure 4a,b show the homogeneous lenses and Figure 4c shows the CSSP lens. The rays of the homogenous spherical lenses are focused near the lens surface and spread out from this position, indicating that ray divergence due to spherical aberration is significant at a longer focal plane in the homogenous spherical lenses. On the other hand, the rays of the CSSP lens are focused slightly further away from the lens surface, and the focused spot size is larger than that of the homogenous lens. However, better ray convergence is observed at a longer focal plane, indicating reduced spherical aberration compared to that of the homogenous lens.

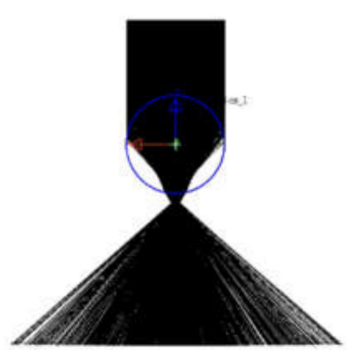

(a)

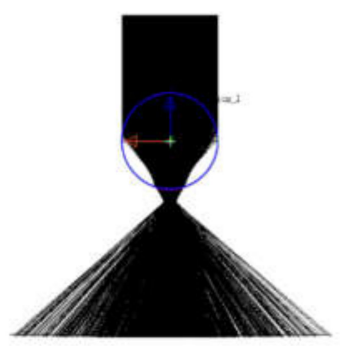

(b)

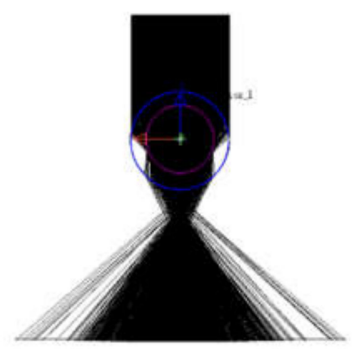

(c)

Figure 4. Ray behavior comparison of the (a) solid plastic (PMMA) homogenous spherical lens, (b) KF-97-500cs homogeneous spherical lens, and (c) CSSP lens.

Figures 5-7 depict the ray distribution, when the plane of the focal length, $f$, is varied for the PMMA homogeneous spherical lens, the KF-96-500cs homogeneous spherical lens, and the CSSP lens, respectively. The $f=15 \mathrm{~mm}$ plane is in contact with the surface of the spherical lens. For both of the homogenous lenses, the smallest spot size appears at the $f=15 \mathrm{~mm}$ plane and increases as $f$ increases. The ray distribution is like that of a Gaussian distribution. For $f \geq 18 \mathrm{~mm}$, the ray convergence degrades. On the other hand, for the CSSP lens, even at $f=24 \mathrm{~mm}$, better concentration is achieved. The ray distribution is similar to a double circle. The outer circle shrinks at $f=24 \mathrm{~mm}$, and both circles merge. 
For $f>24 \mathrm{~mm}$, the ray circle spreads, but the spot is still clearer than that of the homogenous lens at the same $f$.

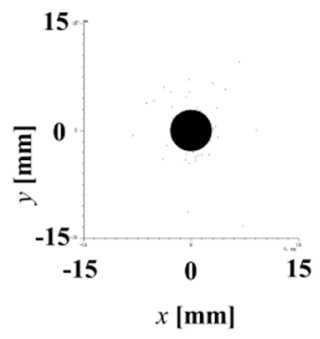

(a)

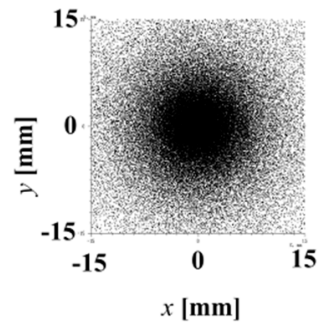

(d)

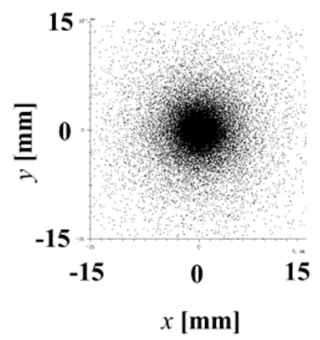

(b)

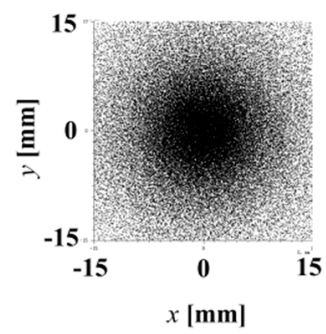

(e)

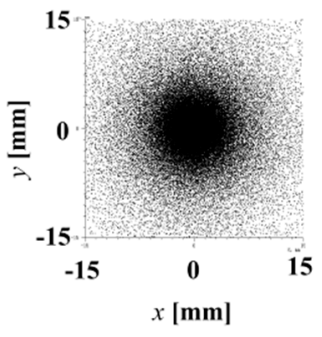

(c)

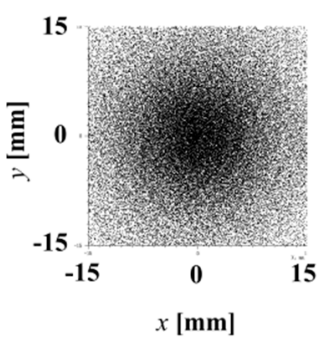

(f)

Figure 5. Simulated ray distribution of the PMMA homogenous spherical lens at the focal plane length $f: f=($ a) $15 \mathrm{~mm},(\mathbf{b}) 18 \mathrm{~mm}$, (c) $24 \mathrm{~mm}$, (d) $30 \mathrm{~mm}$, (e) $36 \mathrm{~mm}$, and (f) $42 \mathrm{~mm}$.

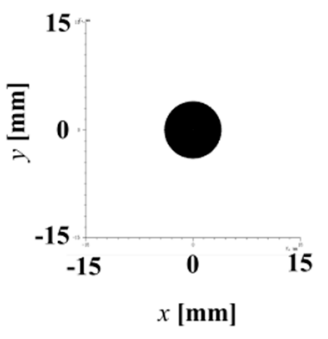

(a)

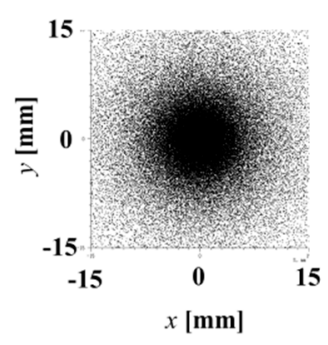

(d)



(b)

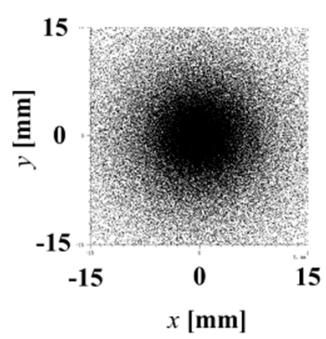

(e)

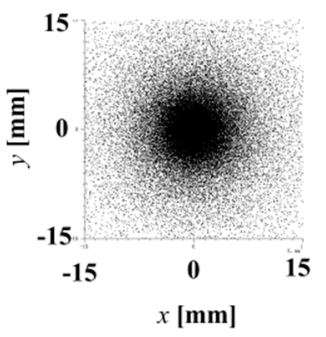

(c)

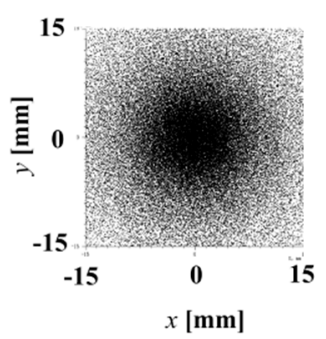

(f)

Figure 6. Simulated ray distribution of the KF-96-500cs homogenous spherical lens at the focal plane length $f: f=$ (a) $15 \mathrm{~mm}$, (b) $18 \mathrm{~mm}$, (c) $24 \mathrm{~mm}$, (d) $30 \mathrm{~mm}$, (e) $36 \mathrm{~mm}$, and (f) $42 \mathrm{~mm}$. 


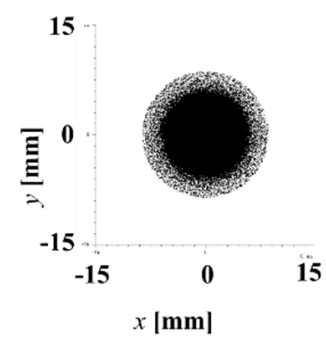

(a)

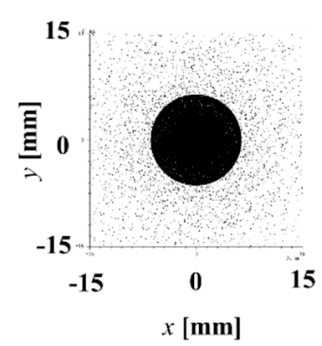

(d)

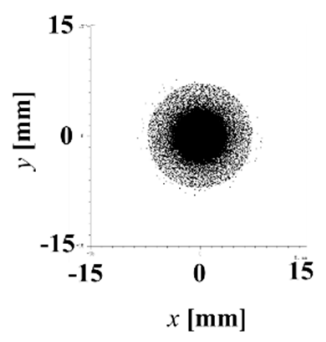

(b)

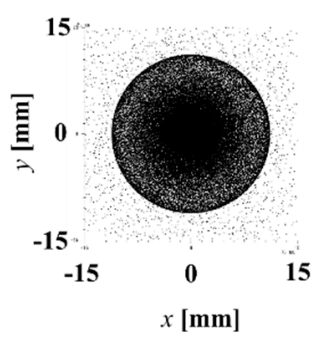

(e)

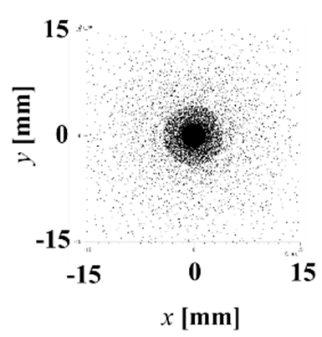

(c)

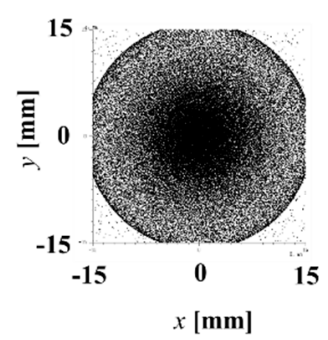

(f)

Figure 7. Simulated ray distribution of the CSSP lens at the focal plane length $f: f=(\mathbf{a}) 15 \mathrm{~mm}$, (b) 18 $\mathrm{mm}$, (c) $24 \mathrm{~mm}$, (d) $30 \mathrm{~mm}$, (e) $36 \mathrm{~mm}$, and (f) $42 \mathrm{~mm}$.

Figure 8 depicts variation in the optical efficiency, $\eta_{\text {opt, }}$ with the change in the focal length, for the CSSP and the homogenous spherical lenses. The simulation conditions are the same as those listed in Table 1. Figure 8a shows the results of a low-concentration condition; the receiver radius, $r_{\text {rec }}=6 \mathrm{~mm}$ and the geometric concentration ratio, $C=6.25 \times$, which is defined as the area ratio of the receiver to the lens aperture. Figure $8 \mathrm{~b}$ shows the results of a high-concentration condition; $\mathrm{r}_{\mathrm{rec}}=1.5 \mathrm{~mm}$ and $C=100 \times$.

In Figure 8a, the focal length range of the PMMA homogenous lens for $\eta_{\text {opt }}>75 \%$ is $f=15-18.3 \mathrm{~mm}$ $(\Delta f=3.3 \mathrm{~mm})$, and that of the KF-96-500cs homogeneous lens is $f=15-23.1 \mathrm{~mm}(\Delta f=8.1 \mathrm{~mm})$, whereas that of the CSSP lens is $f=18.6-27.6 \mathrm{~mm}(\Delta f=9 \mathrm{~mm})$. Thus, in the CSSP lens, a wider focal length range with high optical efficiency exists at a longer focal length position. This characteristic is attributed to the low spherical aberration (as observed in Figure 7), which contributes to widening the mechanical angle limit of the SMTCPV. The homogeneous lenses exhibit a higher $\eta_{\text {opt }}$ at shorter values of $f$ where the beam spot is smaller than the CSSP lens. In Figure $8 \mathbf{b}$, for $\eta_{\text {opt }}>75 \%$, there is no focal length range in the homogenous lenses, while in the CSSP lens, there is a focal length range of $f=21.9-24.6 \mathrm{~mm}(\Delta f=2.7 \mathrm{~mm})$. The peak $\eta_{\text {opt }}$ of the CSSP lens is even higher than that of the homogenous lenses for a higher value of $f$; the PMMA homogeneous lens shows a peak $\eta_{\text {opt }}$ of $73.9 \%$ at $f=17.4 \mathrm{~mm}$; the KF-96-500cs homogeneous lens, $73.8 \%$, at $19.5 \mathrm{~mm}$; the CSSP, $78.1 \%$, at $24.6 \mathrm{~mm}$. These results demonstrate the advantage of the CSSP lens, especially for high concentration conditions. However, even though the volumetric absorption loss by the lens material is ignored, the peak optical efficiency of the CSSP does not reach $80 \%$. The low efficiency is attributed to the losses due to the Fresnel reflection at the Air-PMMA and PMMA-Silicone interfaces, where a part of the rays are directed to the outside of the receiver. The combination of refractive indexes of the lens materials in this study is not always the best; there may be a combination of refractive indexes that can further reduce this loss and improve efficiency. Thus, the best lens material should be further investigated in future work.

Figure 9 shows the simulated $\eta_{\text {opt }}$ of the CSSP lens for varied $r_{\text {core }}$ and $f$ values. The simulation conditions are the same as those listed in Table 1 . Here, $r_{\text {rec }}=1.5 \mathrm{~mm}$ and $C=100 \times$. This result shows that there are two high-optical-efficiency regions around $f=16.5 \mathrm{~mm}$ at $r_{\text {core }}=0-9 \mathrm{~mm}$ and around $f=$ $21 \mathrm{~mm}$ at $r_{\text {core }}=9-15 \mathrm{~mm}$. The latter region shows the highest optical efficiency. This result suggests that the CSSP lens performs better than the homogenous spherical lens at a specific $r_{\text {core }}$ and a longer focal length. 


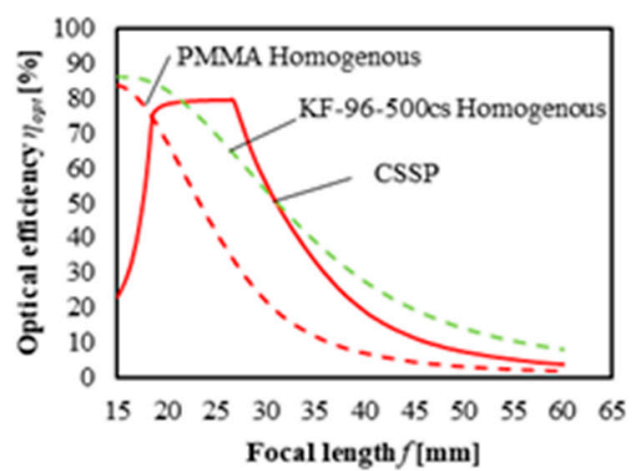

(a)

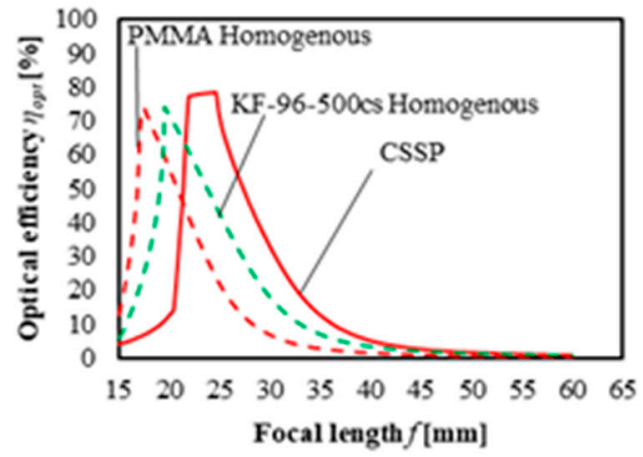

(b)

Figure 8. Simulated optical efficiency across varied focal lengths for the homogenous and core-shell spherical lenses with a the geometric concentration ratio $C$ of (a) $6.25 \times$ and (b) $100 \times$.

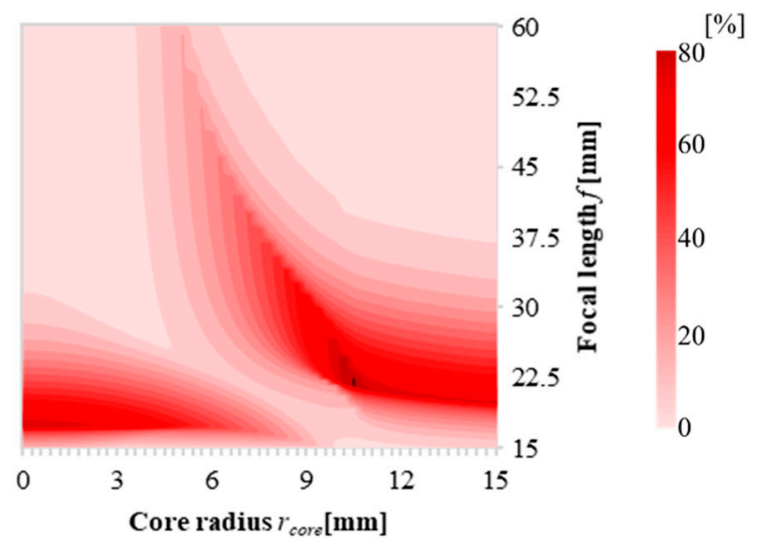

Figure 9. Simulated optical efficiency for varied core radii and focal lengths $(C=100 \times)$.

\subsection{CSSP Lenses Characteristics for Full Solar Spectrum Without Lens Material Absorption}

Prior to the simulation, the wavelength dispersion of RI and the transmittance of KF-96-500cs was measured, as that for PMMA was known that for KF-96-500cs was unknown. RI measurement was performed using an Abbe refractometer DR-M2 (Atago Co., Ltd.). Optical filters for 480, 546, 589, and $656 \mathrm{~nm}$ were used. The measuring temperature was $25.5^{\circ} \mathrm{C} \pm 0.1^{\circ}$. To extrapolate the RI profile for the full solar spectrum range, the measured RIs at the four wavelengths were adopted to the following Cauchy dispersion equation:

$$
n=\alpha_{0}+\frac{\alpha_{2}}{\lambda^{2}}+\frac{\alpha_{4}}{\lambda^{4}}+\ldots+\frac{\alpha_{2 x}}{\lambda^{2 x}}
$$

Here, $n$ is RI at wavelength $\lambda$, and $\alpha_{2 x}$ are the Cauchy coefficients. Table 3 lists the estimated Cauchy coefficients. The absorption measurement was performed using a UV-VIS-IR spectrometer V-670 (JASCO Co., Ltd.). The optical path length was $10 \mathrm{~mm}$. Figure 10 shows the extrapolated profile of RI, $n_{\mathrm{KF}}$ (solid red line), and transmittance, $\% T$ (solid blue line). The measured RIs are illustrated as square points. This result reveals that RI decreases with increasing wavelengths, which is consistent with the reported trend of the conventional silicone elastomer [28]. The transmittance for $\lambda=300-1100 \mathrm{~nm}$ is nearly $100 \%$, and low around $\lambda=1180,1400,1700 \mathrm{~nm}$.

From the wavelength dispersion data of RI, the ray-tracing analysis for chromatic light, i.e., the nearly full solar spectrum, is conducted under the simulation condition B, listed in Table 4 . The power generation performance of a multijunction solar cell decreases when the generated current of each subcell of the multijunction solar cell is different (so-called current mismatch); thus, the effect of chromatic aberration on the concentrated light for spectral bandwidths corresponding to each subcell of the multijunction solar cell is analyzed. 
Table 3. Estimated Cauchy coefficients for KF-96-500cs.

\begin{tabular}{cc}
\hline Cauchy Coefficients & Values \\
\hline$\alpha_{0}$ & 1.389 \\
$\alpha_{2}$ & 0.005296 \\
$\alpha_{4}$ & -0.00018 \\
\hline
\end{tabular}

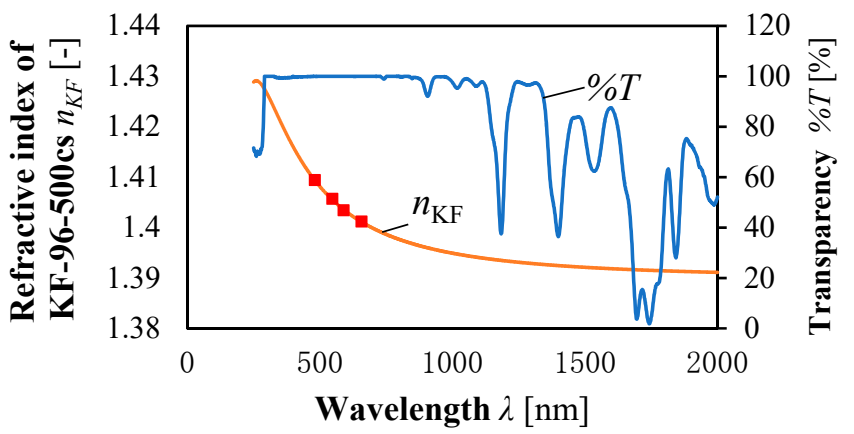

Figure 10. Estimated wavelength dispersion of RI based on the measured RI at four wavelengths and the measured spectral transmittance of KF-96-500cs.

Table 4. Simulation conditions B.

\begin{tabular}{cc}
\hline & CSSP Lens Simulation Specifications \\
\hline Shell RI, $n_{\text {shell }}$ & Wavelength dispersion of PMMA is considered \\
Core RI, $n_{\text {core }}$ & Wavelength dispersion of KF-96-500cs is considered \\
Shell radius, $r_{\text {shell }}$ & $15 \mathrm{~mm}$ \\
Core radius, $r_{\text {core }}$ & $10.2 \mathrm{~mm}\left(0.68 \times r_{\text {shell }}\right)$ \\
Geometrical concentration ratio, $C$ & $100 \times$ \\
Ray spectrum & AM1.5D standard solar spectrum \\
& Top subcell range: $280-749 \mathrm{~nm}$ \\
Absorption by lens materials & Middle subcell range: $750-949 \mathrm{~nm}$ \\
& Bottom subcell range: $950-2000 \mathrm{~nm}$ \\
& Full range: $280-2000 \mathrm{~nm}$ \\
\end{tabular}

Figure 11 shows the simulated $\eta_{\text {opt }}$ of the homogenous and CSSP lenses for varied values of $f$ in comparison with different wavelength bandwidths corresponding to the top, middle, and bottom subcells of the triple-junction solar cell used in the experiment described in the Section 4, as well as the full solar spectrum. Table 5 summarizes the maximum optical efficiency for each spherical lens and wavelength range. As shown in Figure 11a,b, the homogeneous lenses shows sharp peaks at short $f$ and the peak values of the three subcell-ranges are different. This difference is more emphasized in the KF-96-500cs homogeneous lens, and the $\eta_{\text {opt }}$ for the full range is reduced. While in contrast, as shown in Figure 11c, it is confirmed that the $\eta_{\text {opt }}$ of the CSSP lens for the top, middle, and bottom subcells is largely similar in the range of $f=21.9-23.8 \mathrm{~mm}$, where the power generation loss due to the chromatic aberration is minimized and $\eta_{\text {opt }}$ is nearly $80 \%$. For $f<21.9 \mathrm{~mm}, \eta_{\text {opt }}$ of the CSSP lens for the top subcell is greater than that for the middle and bottom subcells; whereas, for $f>23.8 \mathrm{~mm}$, $\eta_{\text {opt }}$ of the CSSP lens for the bottom subcell is greater than that for the middle and top subcells. These characteristics are attributed to the change in the spot size due to chromatic aberration. Compared to Figure $8 \mathbf{b}$, although the chromatic aberration reduces the high- $\eta_{\text {opt }}$ range of $f$, the CSSP lens retains a high $\eta_{\text {opt }}$ at a higher value of $f$ than the homogenous lenses. In usual achromatic lenses, layered structures, e.g., achromatic doublet, reduce the chromatic aberration, however, unlike the spherical lenses, the achromatic lenses must be rotated to keep facing the sun for the solar concentration. 




(a)

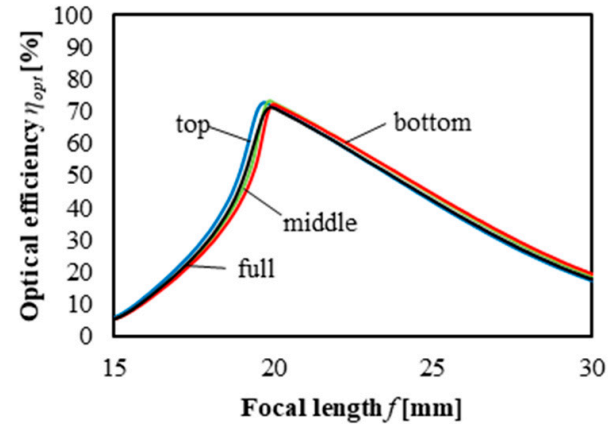

(b)



(c)

Figure 11. Simulated optical efficiency for varied focal lengths in comparison with different wavelength bandwidths corresponding to the top (blue), middle (green), bottom (red) subcell of the triple-junction solar cell, and the full solar spectrum at $C=100 \times$ (black). (a) PMMA homogenous spherical lens, (b) KF-97-500cs homogeneous spherical lens, and (c) CSSP lens.

Table 5. The maximum optical efficiency of the lenses corresponding to Figure 11.

\begin{tabular}{cccc}
\hline Parameters & $\begin{array}{c}\text { PMMA } \\
\text { Homogeneous Lens }\end{array}$ & $\begin{array}{c}\text { KF-96-500cs } \\
\text { Homogeneous Lens }\end{array}$ & Cssp Lens \\
\hline Top subcell range & $74.7 \%$ & $72.9 \%$ & $79.8 \%$ \\
Middle subcell range & $75.2 \%$ & $73.5 \%$ & $79.4 \%$ \\
Bottom subcell range & $75.0 \%$ & $72.4 \%$ & $79.2 \%$ \\
Full range & $74.4 \%$ & $71.5 \%$ & $79.3 \%$ \\
\hline
\end{tabular}

\subsection{CSSP Lenses Characteristics for Full Solar Spectrum with Lens Material Absorption}

Another ray-tracing simulation is performed under the simulation condition $C$ listed in Table 6. Herein, not only the wavelength dispersion of RI but also the spectral transmittance of the lens materials are taken into account; additionally, the effect of the CSSP lens size is analyzed by varying the shell radius from $15-1 \mathrm{~mm}$. To keep the similarity of the lens geometry, the ratio of the core radius to the shell radius is kept at 0.68 .

Figure 12 shows the simulated $\eta_{\text {opt }}$ of the CSSP lens for varied values of $f$ in comparison with different lens sizes when considering the volumetric absorption by the lens materials. On the horizontal axis, $f$ is normalized by the $r_{\text {shell }}$. This result shows that the optical efficiency is improved by reducing the shell radius. For $C=6.25 \times$, the peak $\eta_{\text {opt }}$ percentages are: $71.1 \%$ at $r_{\text {shell }}=15 \mathrm{~mm}, 73.4 \%$ at $10 \mathrm{~mm}, 76.7 \%$ at $5 \mathrm{~mm}$, and $80.0 \%$ at $1 \mathrm{~mm}$; for $C=100 \times, 68.6 \%$ at $15 \mathrm{~mm}, 70.9 \%$ at $10 \mathrm{~mm}, 74.5 \%$ at $5 \mathrm{~mm}$, and $78.1 \%$ at $1 \mathrm{~mm}$. The peak $\eta_{\text {opt }}$ at $r_{\text {shell }}=15 \mathrm{~mm}$ is 8.9 percentage points (at $\left.C=6.25 \times\right)$ and 9.5 percentage point (at $C=100 \times$ ) less than that at $r_{\text {shell }}=1 \mathrm{~mm}$. Thus, the CSSP lenses with smaller radii are more suitable for the SMTCPV. However, because it was difficult to fabricate small lenses, homogenous spherical and CSSP lenses with $r_{\text {shell }}=15 \mathrm{~mm}$ were tested in the experiment presented in the next section. 
Table 6. Simulation conditions C.

\begin{tabular}{cc}
\hline \multicolumn{2}{c}{ CSSP Lens Simulation Specifications } \\
\hline Shell RI, $n_{\text {shell }}$ & Wavelength dispersion of PMMA is considered \\
Core RI, $n_{\text {core }}$ & Wavelength dispersion of KF-96-500cs is considered \\
Shell radius, $r_{\text {shell }}$ & $1,5,10,15 \mathrm{~mm}$ \\
Core radius, $r_{\text {core }}$ & $0.68 \times r_{\text {shell }}$ \\
Geometrical concentration ratio, $C$ & $6.25 \times$ for Figure $12 \mathrm{a}$ \\
Ray spectrum & $100 \times$ for Figure $12 \mathrm{~b}$ \\
& AM1.5D standard solar spectrum \\
Absorption by lens materials & Full range: $280-2000 \mathrm{~nm}$ \\
\hline
\end{tabular}



(a)

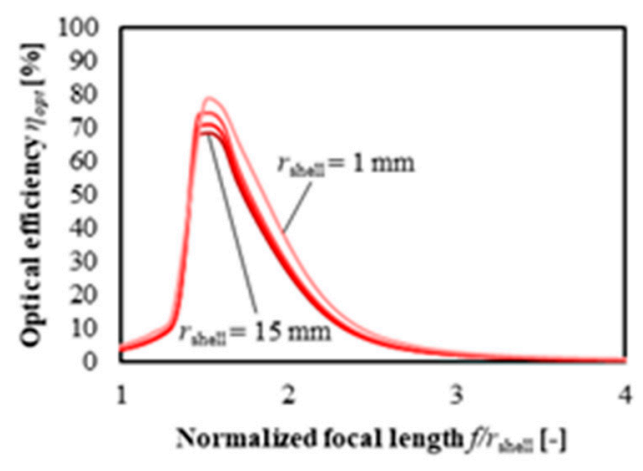

(b)

Figure 12. Simulated optical efficiency of the CSSP lens for varied normalized focal lengths across different shell radii lengths at (a) $C=6.25 \times$ and (b) $100 \times$.

\section{Experiment}

\subsection{Visual Comparison}

Figure 13a,b display comparative photographs of the prototype homogenous and CSSP lenses, respectively (with the structure shown in Figure 3). The $r_{\text {shell }}$ of both lenses was $15 \mathrm{~mm}$. The $r_{\text {core }}$ of the CSSP lens was $9.8 \mathrm{~mm}$. The material of the homogenous lens was PMMA; whereas the materials of the CSSP lens were silicone oil KF-96-500cs (Shin-Etsu chemical) for the core and PMMA for the shell. These photographs were captured when three lines of letters were read the most clearly by the lenses. Comparing Figure 13a with Figure 13b, the letters at the center of both lenses are clear, whereas those at the edge of the homogenous lens are more distorted than those at the edge of the CSSP lens. As spherical aberration occurs at the edge of the spherical lens, the letters are strongly affected, as shown in Figure 13a. On the other hand, Figure 13b shows letters up to the edge of the lens. This result demonstrates that the CSSP lens reduces spherical aberration in the field of view.

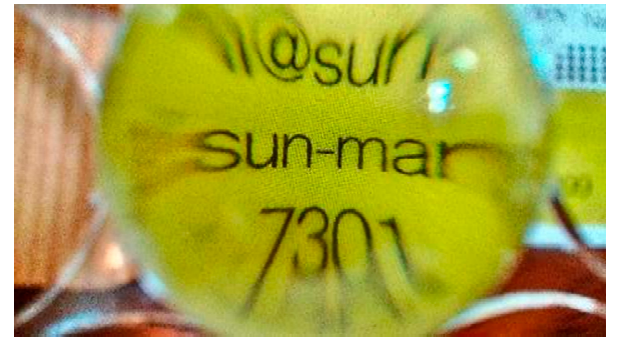

(a)

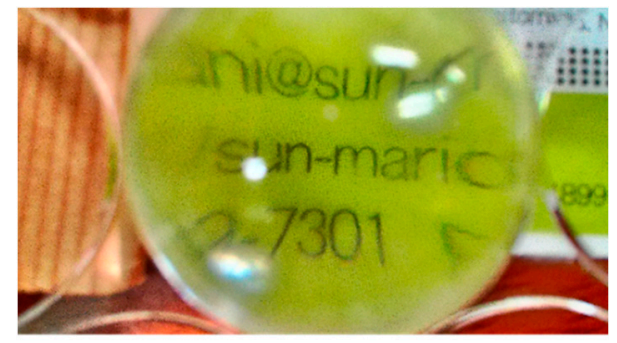

(b)

Figure 13. Comparative photographs of the prototype (a) homogenous spherical lens and (b) CSSP lens. 


\subsection{Outdoor Experiment}

Using the prototype lenses shown in Figure 13, the module efficiency of the lens-cell CPV module was measured under outdoor conditions in Isezaki-city, Japan (latitude $36.1^{\circ}$, longitude $138.6^{\circ}$ ). The direct solar irradiation was measured by a pyrheliometer near the experimental setup (not shown in the figure). Figure 14a shows a solar cell receiver with a lens holder. The spherical lens was attached to this lens holder. By changing the height of the lens holder, the focal length was set as $f=18,24$, and $30 \mathrm{~mm}$, corresponding to the analysis of Figures 5 and 7 . Figure $14 \mathrm{~b}$ shows the lens-cell module with the spherical lens attached to the solar cell receiver; Figure 14c shows a photograph of the measurement setup. The solar cell receiver used was the TCSC100A (Taicrystal Co., Ltd.), with a solar cell area of $100 \mathrm{~mm}^{2}$. The type of solar cell was a GaInP/GaInAs/Ge triple-junction solar cell, which has been designed to achieve approximately $40 \%$ cell efficiency at 500 suns. The lens aperture area was 706.5 $\mathrm{mm}^{2}$, which is the area of a circle with $r_{\text {shell }}=15 \mathrm{~mm}$; hence, $C=7 \times$. It is noted that this concentration ratio is much lower that of the designed concentration ratio of the solar cell used in the experiment. It is also noted that in this experiment, the solar cell can receive the light impinging directly to the solar cell (without going through the optics), which is mostly a part of the diffuse component of sunlight, and is very small compared to the concentrated direct component; thus, it does not have a significant impact on changing the trend of the results.

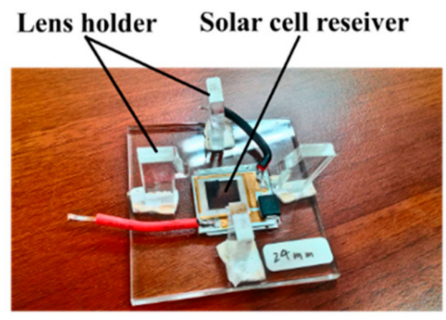

(a)

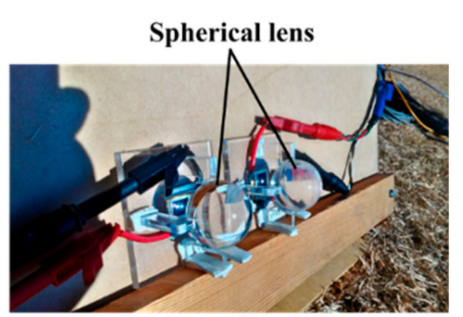

(b)

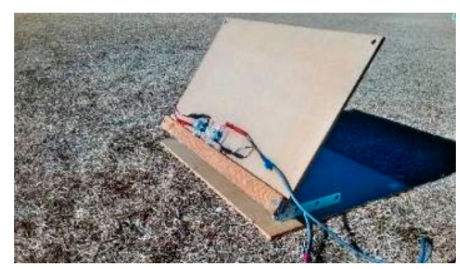

(c)

Figure 14. Test module photographs: (a) Solar cell receiver, (b) lens-cell module, and (c) measurement setup.

Figure 15 shows photographs of concentrated sunlight at the surface of the solar cell for both prototype lenses with different focal lengths. For the homogenous spherical lens, the light spot is concentrated sufficiently for $f=18 \mathrm{~mm}$ (left of Figure 15a); the light spot was distorted because of spherical aberration for $f=24 \mathrm{~mm}$ (left of Figure 15b); the light spot cannot be seen for $f=30 \mathrm{~mm}$ (left of Figure 15c). For the CSSP lens, a large light spot can be seen for $f=18 \mathrm{~mm}$ (right of Figure 15a); the light spot becomes smaller for $f=24 \mathrm{~mm}$ (right of Figure 15b). The light spot is still maintained because of low aberration for $f=30 \mathrm{~mm}$ (right of Figure 15c). The behavior of the light spot is consistent with that of the simulated result in Figures 5 and 7. Under the condition of $C=100 \times$ in Figure $8 b$, the homogenous lens with $f=18 \mathrm{~mm}$ and the CSSP lens with $f=24 \mathrm{~mm}$ achieve high optical efficiency. These high optical efficiency conditions correspond to the conditions of the figure, where the light spot is smaller and shrunk (left of Figure 15a and right of Figure 15b).

Figure 16 shows the short circuit current, $I_{\mathrm{sc}}$, and the module efficiency, $\eta_{\text {module, }}$ for the homogenous and CSSP lenses. The dashed line denotes the homogenous lens, whereas the solid line denotes the CSSP lens. Current-voltage curves were measured every $20 \mathrm{~s}$ under constant direct solar irradiation and $I_{\mathrm{sc}}$ 
open circuit voltage, fill factor, and maximum power were obtained by averaging the measured data over 10 minutes. The $\eta_{\text {module }}$ was then calculated as the ratio of maximum power to the direct solar irradiation incident on the lens aperture area. The results for $f=18,24$, and $30 \mathrm{~mm}$ were obtained under no-cloud condition at 11:03-11:14, 13:27-13:37, and 11:21-11:31, respectively. Though the solar spectrum was not directly measured, around noon on a clear sky day, the spectrum is generally neither too red-shifted nor too blue-shifted, according to long-term observation data of solar spectrums in Japan [29].
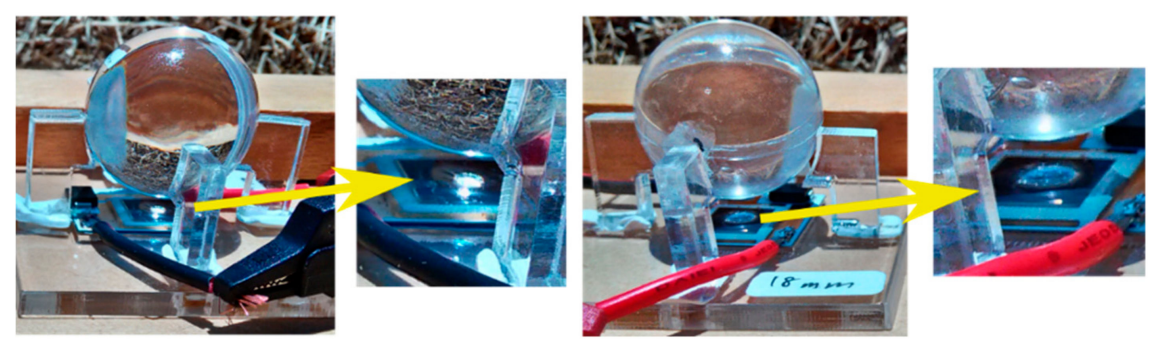

(a)
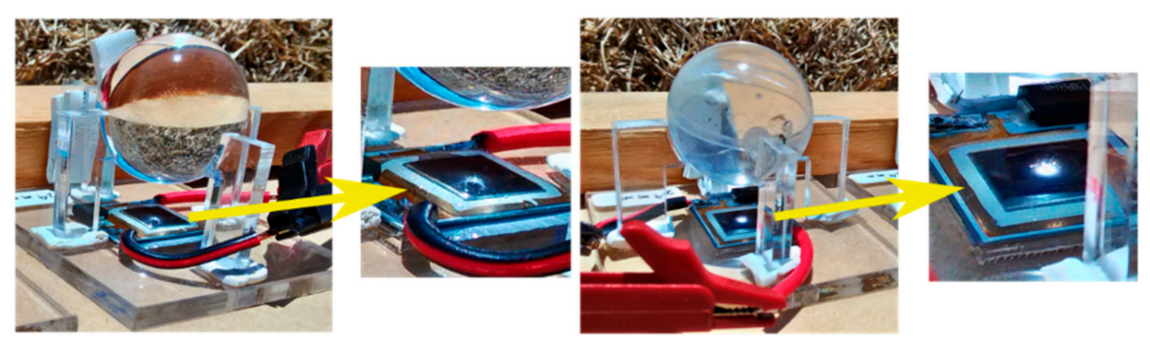

(b)
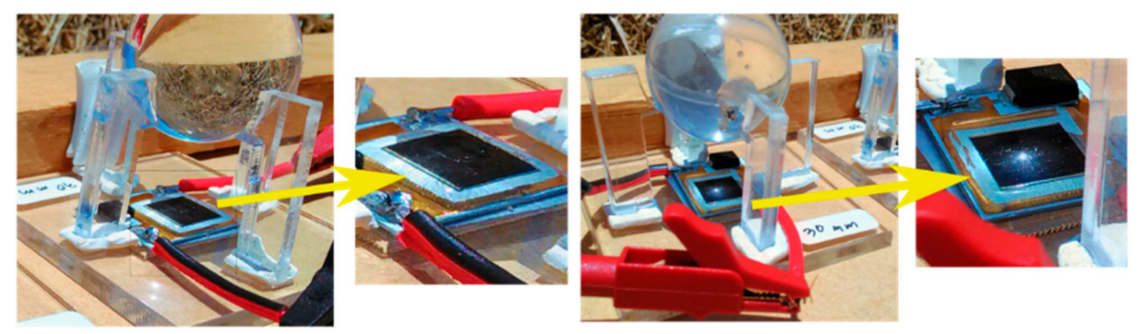

(c)

Figure 15. Photographs of concentrated sunlight by the homogenous (left) and the CSSP lenses (right) with different focal lengths $f: f=$ (a) $18 \mathrm{~mm}$, (b) $24 \mathrm{~mm}$, and (c) $30 \mathrm{~mm}$.

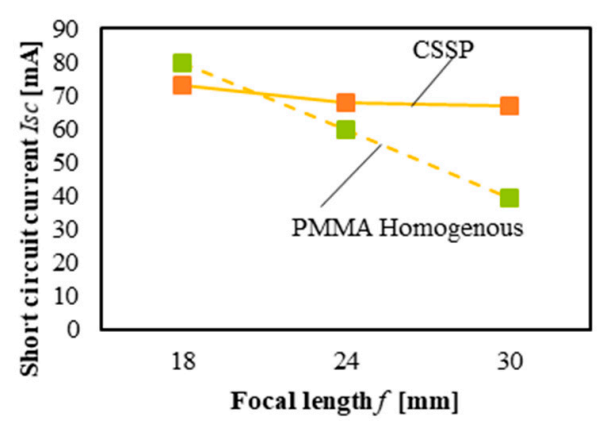

(a)

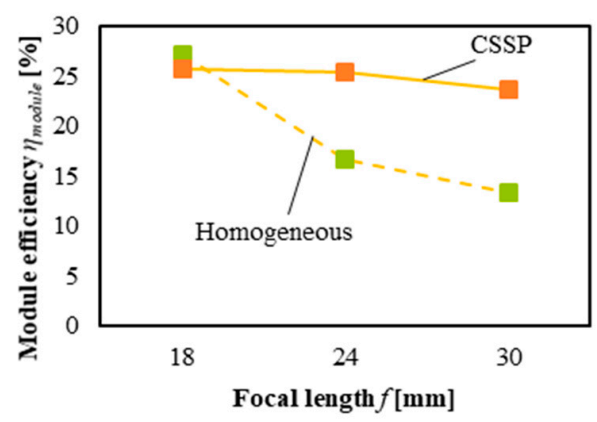

(b)

Figure 16. Experimental results of the lens-cell module for the homogenous and CSSP lenses regarding the (a) short circuit current and (b) module efficiency. 
It was confirmed that the lens-cell module with the CSSP lens exhibited greater $I_{\mathrm{sc}}$ and $\eta_{\text {module }}$ than that with the homogenous lens for a longer $f$. This trend was more emphasized in the $\eta_{\text {module }}$. This is because, with the homogenous lens, not only $I_{\mathrm{sc}}$ but also the fill factor decreases as $f$ increases. The fill factor decreased because of non-uniformity of concentrated sunlight at the solar cell surface, as shown in the left side of Figure 15b. The resulting $I_{\mathrm{sc}}$ and $\eta_{\text {module }}$ of the homogenous lens at $f=18 \mathrm{~mm}$ were high: $I_{\mathrm{sc}}=79.5 \mathrm{~mA}$ and $\eta_{\text {module }}=27.1 \%$. However, as $f$ increased, $I_{\mathrm{sc}}$ and $\eta_{\text {module }}$ decreased drastically. On the other hand, for the CSSP lens, the results for $f=18,24$, and $30 \mathrm{~mm}$ were $I_{\mathrm{sc}}=$ $73.3 \mathrm{~mA}$ and $\eta_{\text {module }}=25.7 \%, I_{\mathrm{sc}}=68.0 \mathrm{~mA}$ and $\eta_{\text {module }}=25.4 \%$, and $I_{\mathrm{sc}}=67.1 \mathrm{~mA}$ and $\eta_{\text {module }}=$ $23.6 \%$, respectively. Even when $f$ increased further, $I_{\mathrm{sc}}$ and $\eta_{\text {module }}$ decreased slightly.

A previous study [23] presented the relationship between the normalized focal length, $f / r_{\text {shell }}$, and the mechanical limit of the tracking angle, $\theta_{\text {in }}$. The results for $f=18,24$, and $30 \mathrm{~mm}$ were $\theta_{\text {in }}=33.6^{\circ}$, $51.3^{\circ}$, and $60^{\circ}$, respectively. A high module efficiency at a long focal length leads to an increase in $\theta_{\text {in }}$ in the SMTCPV system.

Figure 17 shows a comparison between the measured $I_{\mathrm{sc}}$ values from Figure 16 and the simulated $\eta_{\mathrm{opt}}$ of Figure 8a. The $I_{\mathrm{sc}}$ of the solar cell is generally proportional to irradiation at the solar cell surface. Thus, $I_{\mathrm{sc}}$ is considered a measure of the optical efficiency of the concentrator. The red line indicates the simulated $\eta_{\mathrm{opt}}$, while the orange line indicates the measured $I_{\mathrm{sc}}$. The dashed line indicates the homogenous spherical lens, whereas the solid line indicates the CSSP lens. The orange points indicate the actual measuring points. The horizontal axis is the normalized focal length $f / r_{\text {shell }}$. This result shows that the tendency of the measured $I_{\mathrm{sc}}$ is qualitatively consistent with the simulated $\eta_{\mathrm{opt}}$. The trend of $I_{\mathrm{sc}}$ of the CSSP lens was lower compared to that of the simulated $\eta_{\mathrm{opt}}$; this is likely caused by the occurrence of stray light at the bonding part of the upper and bottom parts of the shell. Otherwise, lens shape error may have occurred in the lens fabrication process. A higher concentration experiment using a smaller triple-junction solar cell was also attempted; however, sharp focusing and the concentration performances were very poor. This may also be attributed to lens shape error during fabrication and/or deformation during experimentation. By further identifying reasons for the lower $I_{\mathrm{sc}}$ of the CSSP lens compared to that of the simulated $\eta_{\mathrm{opt}}, I_{\mathrm{sc}}$ and $\eta_{\text {module }}$ can be increased further. Usage of a solar cell designed for a proper concentration ratio nearer to the experimental condition can also increase $\eta_{\text {module }}$.

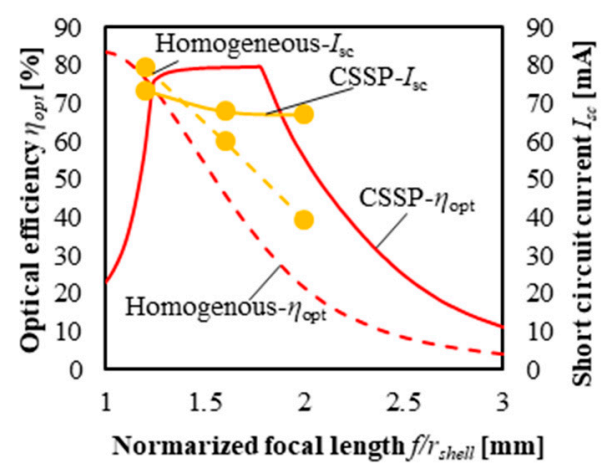

Figure 17. Comparison of the measured short circuit current and the simulated optical efficiency.

\section{Conclusions}

The optical characteristics of a radially symmetrical CSSP lens is analyzed regarding its suitability to microtracking CPV applications. The CSSP lens, with a core composed of a material with a lower refractive index, exhibits a higher optical efficiency than the homogenous spherical lens for longer focal lengths. The CSSP lens with the appropriate core radius can reduce the spherical and chromatic aberrations for a long focal length. This characteristic is preferable for microtracking CPVs with a spherical lens array to achieve a higher module efficiency for a wider incidence angle. In the outdoor experiment, the lens-cell CPV module with the prototype CSSP lens made of commercially 
available materials showed superior optical efficiency and module efficiency compared to the module with the homogenous spherical lens, as expected by the optical simulation. Because the simulated and experimental conditions were limited to the on-axis condition, i.e., an incidence angle of zero, and a single lens, further investigations are necessary to determine the practical characteristics and performance of the microtracking CPV system with the CSSP lens array. Additionally, the lower optical performance of the fabricated CSSP lens compared to the simulated one should be further investigated. Thus, a manufacturing method to fabricate the CSSP lens without optical defects is also necessary, as well as the selection of appropriate core and shell materials.

Author Contributions: Conceptualization, M.N.; formal analysis, M.N.; experimentation, M.N.; writing—original draft preparation, M.N.; writing—review and editing, N.Y.; supervision, N.Y.

Funding: This research received no external funding.

Conflicts of Interest: The authors declare no conflict of interest.

\section{References}

1. Dimroth, D.F. New World Record for Solar Cell Efficiency at 46\%-French-German Cooperation Confirms Competitive Advantage of European Photovoltaic Industry. Available online: https://www.ise.fraunhofer.de/ en/press-media/press-releases/2014/new-world-record-for-solar-cell-efficiency-at-46-percent.html (accessed on 11 September 2019).

2. Steiner, M.; Bösch, A.; Dilger, A.; Dimroth, F.; Dörsam, T.; Muller, M.; Hornung, T.; Siefer, G.; Wiesenfarth, M.; Bett, A.W. FLATCON ${ }^{\circledR}$ CPV module with $36.7 \%$ efficiency equipped with four-junction solar cells. Prog. Photovolt. Res. Appl. 2015, 23, 1323-1329. [CrossRef]

3. Apostoleris, H.; Stefancich, M.; Chiesa, M. Tracking-integrated systems for concentrating photovoltaics. Nat. Energy 2016, 1, 16018. [CrossRef]

4. Duerr, F.; Meuret, Y.; Thienpont, H. Tailored free-form optics with movement to integrate tracking in concentrating photovoltaics. Opt. Express 2013, 21, A401-A411. [CrossRef]

5. Zagolla, V.; Dominé, D.; Tremblay, E.; Moser, C. Self-tracking solar concentrator with an acceptance angle of $32^{\circ}$. Opt. Express 2014, 22, A1880-A1894. [CrossRef]

6. $\mathrm{Ma}, \mathrm{H} . ; \mathrm{Wu}, \mathrm{L}$. Horizontally staggered lightguide solar concentrator with lateral displacement tracking for high concentration applications. Appl. Opt. 2015, 54, 6217-6223. [CrossRef]

7. Michel, C.; Blain, P.; Clermont, L.; Languy, F.; Lenaerts, C.; Fleury-Frenette, K.; Décultot, M.; Habraken, S.; Vandormael, D.; Cloots, R.; et al. Waveguide solar concentrator design with spectrally separated light. Sol. Energy 2017, 157, 1005-1016. [CrossRef]

8. Hallas, J.M.; Baker, K.A.; Karp, J.H.; Tremblay, E.J.; Ford, J.E. Two-axis solar tracking accomplished through small lateral translations. Appl. Opt. 2012, 51, 6117-6124. [CrossRef]

9. Dhakal, R.; Lee, J.; Kim, J. Bio-inspired thin and flat solar concentrator for efficient, wide acceptance angle light collection. Appl. Opt. 2014, 53, 306-315. [CrossRef]

10. Karp, J.H.; Tremblay, E.J.; Hallas, J.M.; Ford, J.E. Orthogonal and secondary concentration in planar micro-optic solar collectors. Opt. Express 2011, 19, A673-A685. [CrossRef]

11. Bouchard, S.; Thibault, S. Planar waveguide concentrator used with a single axis tracker. Opt. Express 2014, 51, 6848-6854. [CrossRef]

12. Price, J.S.; Sheng, X.; Meulblok, B.M.; Rogers, J.A.; Giebink, N.C. Wide-angle planar microtracking for quasi-static microcell concentrating photovoltaics. Nat. Commun. 2015, 6, 6223. [CrossRef]

13. Lloyd, J.; Pavilonis, M.; Gladden, C.; Casper, C.; Schneider, K.; McMahon, W. Performance of a prototype stationary catadioptric concentrating photovoltaic module. Opt. Express 2018, 26, A413-A419. [CrossRef]

14. Vu, N.; Shin, S. Flat Concentrator Photovoltaic System with Lateral Displacement Tracking for Residential Rooftops. Energies 2018, 11, 114. [CrossRef]

15. Ito, A.; Sato, D.; Yamada, N. Optical design and demonstration of microtracking CPV module with bi-convex aspheric lens array. Opt. Express 2018, 26, A879-A891. [CrossRef]

16. Schrank, H.; Sanford, J. A Luneberg-Lens Update. IEEE Antennas Propag. Mag. 1995, 37, 76-79.

17. Morgan, S.P. General solution of the Luneberg lens problem. J. Appl. Phys. 1958, 29, 1358-1368. [CrossRef] 
18. Kotsidas, P.; Modi, V.; Gordon, J.M. Realizable planar gradient-index solar lenses. Opt. Lett. 2012, 37, 1235-1237. [CrossRef]

19. Dodge, M.J. Refractive properties of magnesium fluoride. Appl. Opt. 1984, 23, 1980-1985. [CrossRef]

20. Liu, Z.; Yang, S.; Nie, Z. A dielectric lens antenna design by using the effective medium theories. In Proceedings of the 2010 International Symposium on Intelligent Signal Processing Communication Systems, Chengdu, China, 6-8 December 2010; pp. 1-4.

21. Xi, J.Q.; Schubert, M.F.; Kim, J.K.; Schubert, E.F.; Chen, M.; Lin, S.Y.; Liu, W.; Smart, J.A. Optical thin-film materials with low refractive index for broadband elimination of Fresnel reflection. Nat. Photonics 2007, 1, 176-179. [CrossRef]

22. Tabata, M.; Adachi, I.; Ishii, Y.; Kawai, H.; Sumiyoshi, T.; Yokogawa, H. Development of transparent silica aerogel over a wide range of densities. Nucl. Instrum. Methods Phys. Res. A 2010, 623, 339-341. [CrossRef]

23. Nakatani, M.; Yamada, N. Optical Simulation of Two-Shell Spherical Lens for Microtracking CPV System. In Proceedings of the IEEE 7th World Conference on Photovoltaic Energy Conversion, Waikoloa, HI, USA, 10-15 June 2018; pp. 927-930.

24. Rhys, T.L. The Design of Radially Symmetric Lenses. IEEE Trans. Antennas Propag. 1970, 18, 497-506. [CrossRef]

25. Kotsidas, P.; Modi, V.; Gordon, J.M. Nominally stationary high-concentration solar optics by gradient-index lenses. Opt. Express 2011, 19, 2325-2334. [CrossRef]

26. Shin-Etsu-Chemical Silicone Fluid Catalog Shin-Etsu Chemical Catalog. Available online: http://www. shinetsusilicone-global.com/catalog/pdf/fluid_e.pdf (accessed on 11 September 2019).

27. Sultanova, N.; Kasarova, S.; Nikolov, I. Dispersion properties of optical polymers. Acta Phys. Pol. A 2009, 116, 585-587. [CrossRef]

28. Cooper, P.R. Refractive-index measurements of paraffin, a silicone elastomer, and an epoxy resin over the 500-1500-nm spectral range. Appl. Opt. 1982, 21, 3413-3415. [CrossRef]

29. New Energy and Industrial Technology Development (NEDO), Solar Spectrol Database VER-3 2018. Available online: https://www.nedo.go.jp/library/nissharyou.html (accessed on 11 September 2019).

(C) 2019 by the authors. Licensee MDPI, Basel, Switzerland. This article is an open access article distributed under the terms and conditions of the Creative Commons Attribution (CC BY) license (http://creativecommons.org/licenses/by/4.0/). 